\title{
Evaluation of an automated knowledge based treatment planning system for head and neck
}

\author{
Jerome Krayenbuehl ${ }^{*}$, lan Norton ${ }^{2}$, Gabriela Studer $^{1}$ and Matthias Guckenberger ${ }^{1}$
}

\begin{abstract}
Background: This study evaluated an automated inverse treatment planning algorithm, Pinnacle Auto-Planning (AP), and compared automatically generated plans with historical plans in a large cohort of head and neck cancer patients.

Methods: Fifty consecutive patients treated with volumetric modulated arc therapy (Eclipse, Varian Medical System, Palo Alto, CA) for head and neck were re-planned with AP version 9.10. Only one single cycle of plan optimization using one single template was allowed for AP. The dose to the planning target volumes (PTV's; 3-4 dose levels), the organs at risk (OAR's) and the effective working time for planning was evaluated. Additionally, two experienced radiation oncologists blind-reviewed and ranked 10 plans.

Results: Dose coverage and dose homogeneity of the PTV were significantly improved with AP, however manually optimized plans showed significantly improved dose conformity. The mean dose to the parotid glands, oral mucosa, swallowing muscles, dorsal neck tissue and maximal dose to the spinal cord were significantly reduced with AP. In $64 \%$ of the plans, the mean dose to any OAR (spinal cord excluded) was reduced by $>20 \%$ with AP in comparison to the manually optimized plans. In $12 \%$ of the plans, the manually optimized plans showed reduced doses by $>20 \%$ in at least one OAR. The experienced radiation oncologists preferred the AP plan and the clinical plan in 80 and $20 \%$ of the cases, respectively. The average effective working time was $3.8 \mathrm{~min} \pm 1.1 \mathrm{~min}$ in comparison to $48.5 \mathrm{~min} \pm 6.0$ min using AP compared to the manually optimized plans, respectively.

Conclusion: The evaluated automated planning algorithm achieved highly consistent and significantly improved treatment plans with potentially clinically relevant OAR sparing by $>20 \%$ in $64 \%$ of the cases. The effective working time was substantially reduced with Auto-Planning.
\end{abstract}

Keywords: Volumetric modulated arc therapy, Head and neck, Automated planning optimization

\section{Introduction}

Intensity modulated radiotherapy (IMRT) and volumetric modulated radiotherapy (VMAT) have been used for more than a decade and are now standard techniques for external beam radiotherapy treatment (RT). However, the inverse and computer-based planning approach involves multiple manual steps, which might influence the plan quality and consistency: planning objectives and constraints need to be manually adapted to the patient

\footnotetext{
* Correspondence: Jerome.krayenbuehl@usz.ch

${ }^{1}$ Department of Radiation Oncology, University Hospital Zurich, Klinik für Radio-Onkologie, Rämistrasse 100, CH-8091 Zürich, Switzerland

Full list of author information is available at the end of the article
}

individual anatomy and tumor location, size and shape [1]. Additional help structures are frequently defined to further individualize and optimize the treatment plan on a patient individual basis resulting in an iterative process of IMRT and VMAT plan generation. Furthermore, the TPS operator needs to have profound knowledge and experience about the limitations of the treatment planning system and techniques, translating this into a prediction of the dose distribution, which can be achieved in each individual case. This method of manual optimization is time consuming, especially for complex cases such as head and neck carcinoma where multiple

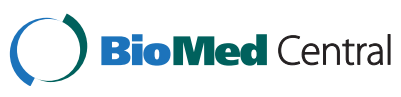

(c) 2015 Krayenbuehl et al. Open Access This article is distributed under the terms of the Creative Commons Attribution 4.0 International License (http://creativecommons.org/licenses/by/4.0/), which permits unrestricted use, distribution, and reproduction in any medium, provided you give appropriate credit to the original author(s) and the source, provide a link to the Creative Commons license, and indicate if changes were made. The Creative Commons Public Domain Dedication waiver (http://creativecommons.org/publicdomain/zero/1.0/) applies to the data made available in this article, unless otherwise stated. 
target dose levels are defined in close proximity to OAR's.

In order to improve the overall plan quality and consistency, and to decrease the time required for planning, semi-automated planning algorithms have been developed $[2,3]$. In the present study, we have evaluated a fully automated treatment planning system (TPS), Pinnacle Auto-Planning (Philips Radiation Oncology Systems), which uses an iterative algorithm based approach to automatically adapt objectives, constraints and dose shaping contours during the optimization process to achieve clinical goals. It was the aim of this study to evaluate Auto-Planning in a large cohort of head and neck cancer patients: treatment plans were compared with historical, clinically accepted VMAT treatment plans generated by Eclipse RapidArc planning (Varian Medical System, Palo Alto, CA). Additionally, time required for manual vs. Auto-Planning was compared.

\section{Methods and materials} Automatic VMAT optimization

A new optimizer, Auto-Planning, was introduced in version 9.10 with the Pinnacle TPS (Philips Radiation Oncology Systems, Fitchburg, WI). It is a fully integrated module in the TPS, similar to the "manual" inverse optimizer module. Auto-Planning employs an iterative algorithm approach to reach and potentially surpass user defined clinical goals. During Auto-Planning, individual optimization goals, constraints and weights are automatically added and adjusted. The IMRT/VMAT optimizer is automatically run multiple times with adjustments being made during and between optimization runs. Additionally, Auto-Planning adjusts the priority of clinical goals based on their probability of being achieved. Therefore, Auto-Planning tries to mimic the decision making process of an experienced TPS operator. In addition to clinical objectives and priorities, AutoPlanning has a compromise setting to allow for sparing of serial organs such as the spinal cord over targets, and advanced settings allow the user to set global parameters such as priorities between targets and OAR's, dose falloff, maximum dose and cold spot management.

\section{Ethics approval and patient selection}

All patients included into this study have given their approval to use their data for scientific research.

For this retrospective treatment planning study, 50 consecutive patients with diagnosis of primary mesopharynx, hypopharynx, oropharynx or larynx carcinoma patients treated in our department were enrolled. Each patient was treated with at least three dose levels: 70Gy or $69.63 \mathrm{~Gy}, 60 \mathrm{~Gy}$ and $54 \mathrm{~Gy}$ within 35 or 33 fractions using a simultaneous integrated boost concept. Dose levels of 66Gy and 52Gy were additionally defined for six and 11 patients, respectively. The dose was normalized to the mean target dose of the highest-order planning target volume (PTV). The following critical structures were analyzed: parotids, spinal cord, brain stem, mandibular bones, oral mucosa, pharynx, dorsal neck tissue.

All patients were treated with the VMAT technique using two $360^{\circ}$ - arcs. Manual VMAT treatment planning was performed using RapidArc planning in Eclipse treatment planning version 11.0.42. The treated plans were manually optimized for a 6-MV photon linear accelerator (Trilogy, TrueBeam or Edge; Varian Medical Systems). Clinically accepted and delivered treatment plans served as reference in this study. All plans were generated by experienced dosimetrists according to written protocols. All treatment plans were verified by a medical physicist and two radiation oncologist physicians before treatment for all cases.

\section{Retrospective planning study using Auto-Planning}

Plans optimized with Auto-Planning utilized a single model where clinical objectives and priorities for each PTV and OAR are defined. The constraints for the target consist of a maximum dose allowed and a dose level to be achieved which corresponds to the dose to $98 \%$ of the target volume. The clinical objectives for an OAR include dose-volume histogram, maximum dose and mean dose. Four priority levels can be defined for the OAR: low, medium, high or constrain. Depending on the patient-individual overlap of the OAR with the targets, the priority can be automatically adjusted from high to medium ( $>25 \%$ overlapping), or to low ( $>50 \%$ overlapping). It is possible for the user to over-ride automatic adjustment of individual priorities, but this is outside the scope of this investigation. In addition to priority, there is an additional compromise setting, which allows for individual OAR priority over targets (such as for spinal cord).

A model (pre-set of Auto-planning preferences and target \& OAR objectives) for head and neck planning was created in Auto-Planning based on five head and neck cases. These five cases were not included in the set of patients used for the plan comparison. The AutoPlanning model was optimized by adjusting the clinical objectives and priorities until all five plans satisfied clinical target and organ at risk goals. All study plans were then optimized with the same model. The beam geometry for Auto-Planning was similar to the clinical plans using two $360^{\circ}$-arcs with collimator set to $5^{\circ}$ and $355^{\circ}$. Ninety control points per arc were set for the AutoPlanning plans and 177 for the clinical plans. The ability to make individual adjustments to the model prior to running Auto-Planning was limited to the target doses in this study and was not allowed for OARs. 
One treatment plan was generated for each patient using Auto-planning and only one optimization cycle was allowed. This plan was used for comparison with historical treatment plans.

Quality assurance for each clinical plan and for ten Auto-Planning plans was performed on a phantom (Delta4, ScandiDos AB, Uppsala, Sweden). All fields had to have a gamma value $>95 \%$ with a distance to agreement of $3 \mathrm{~mm}$ and a dose difference of $3 \%$.

\section{Plan comparison}

Dose-volume histograms (DVHs) were calculated for the PTVs and OARs for each plan. For comparison purposes, DVHs were normalized to the mean dose of the high dose PTV (70Gy or 69.63Gy over 35 or 33 fractions). Target dose distribution was evaluated according to the Paddick conformity index (CI) [4], the target coverage defined as the volume enclosed by the $95 \%$ isodose line and the dose homogeneity index (HI) [1] defined as the ratio between the dose covering $5 \%$ of the PTV volume to the dose covering $95 \%$ of the PTV volume.

The dose to OARs was evaluated according to the mean dose for all OARs except for the spinal cord, where the maximum dose to $0.1 \mathrm{~cm}^{3}$ was evaluated.

In addition to DVH parameters evaluation, two experienced radiation oncologists blind-reviewed and ranked 10 randomized plans. They had to choose which plan they preferred.

\section{Planning time}

The working time required by one highly experienced dosimetrist to generate a plan was measured for the last ten head and neck patients treated in our department. The time was measured for the manual optimized plans and for Auto-Planning. The planning time was defined as the effective working time required between the point where the target and OAR volumes are defined by the clinicians to the time were the plans is accepted by responsible clinicians. This included the time needed for the definition of the help structures and to re-adjust the constraints and help structures after each optimization. The time required for the optimization was not measured as it depends strongly on the number of plans running in parallel on the server.

\section{Statistics}

Statistical analysis was performed using a paired $t$ test. A $\mathrm{p}$ value of $<0.05$ was accepted as significant.

\section{Results}

OAR objectives and priorities used for Auto-Planning are listed in Table 1. These settings were used for the
Table 1 Dose objectives used in Auto-Planning for the optimization of head and neck plans

\begin{tabular}{lll}
\hline & Constraint & Priority \\
\hline Myelon & Dmax $<43 G y$ & Medium \\
Brainstem & Dmax $<$ 48Gy & Medium \\
Parotid & Mean dose $<22 G y$ & High \\
Parotid & Mean dose $<$ 15Gy & Medium \\
Oral mucosa & Mean dose $<$ 20Gy & High \\
Oral mucosa & Mean dose $<$ 12Gy & Low \\
Pharynx & Dmax $<45 G y$ & Medium \\
Pharynx & Mean dose $<20 G y$ & High \\
Pharynx & Mean dose $<$ 15Gy & Medium \\
Plexus & Dmax $<61 G y$ & Medium \\
Mandibular bones & Dmax $<60 G y$ & Medium \\
Mandibular bones & Mean dose $<22 G y$ & Medium \\
Brain & Dmax $<48 G y$ & Medium \\
Brain & Mean dose $<20 G y$ & Medium \\
Dorsal neck tissue & Mean dose $<25 G y$ & Medium \\
\hline
\end{tabular}

optimization of all head-and-neck cases planned with Auto-Planning. No individualization was performed.

\section{Target volumes}

The average DVH parameters for the clinical and AutoPlanning plans for targets and OARs are shown in Table 2. The plans were normalized to the mean dose of the high dose PTV. Differences in dose homogeneity (HI) reached significance only for PTV 70Gy: HI was $1.066 \pm 0.015$ for Auto-Planning and $1.091 \pm 0.017$ for the clinical plans $(p<0.001)$. For PTV 60Gy and PTV 54Gy, HI was $1.098 \pm 0.028$ and $1.107 \pm 0.018$ for the Auto-planning plans and $1.103 \pm 0.025$ and $1.106 \pm 0.021$ for the clinical plans, $p=0.06$ and $\mathrm{p}=0.28$.

The target coverage was significantly improved with Auto-Planning by 2.9, 3.0 and $1.3 \%$ for the PTV 70Gy, PTV 60Gy and PTV 54Gy $(p<0.01)$. However, the CI decreased significantly with Auto-Planning $(p<0.001)$ for the PTV 70Gy and for the PTV from 0.93 to 0.86 and from 0.68 to 0.64 .

\section{Organs at risk}

Comparison of OAR sparing is summarized in Table 2. Both Auto-Planning and clinical plans were able to keep the maximum spinal cord dose below our tolerance of 45Gy for each patient. Auto-Planning further reduced the maximal dose to the spinal cord by $1.6 \mathrm{~Gy}$ in comparison to the clinical plans $(p<0.01)$.

The mean doses to the ipsilateral and contralateral parotid were reduced with Auto-Planning by $1.9 \mathrm{~Gy}$ and 2.0 Gy respectively $(p<0.01)$, see Table 2 . The largest reduction of the mean parotid dose with Auto-Planning 
Table 2 Dose-volume histogram parameters: comparison of clinical and Auto-Planning plans

\begin{tabular}{|c|c|c|c|c|c|}
\hline & \multicolumn{2}{|c|}{ Clinical plan } & \multicolumn{2}{|c|}{ AutoPlanning } & \multirow[t]{2}{*}{$t$-test } \\
\hline & Mean & $\overline{S t D e v}$ & Mean & StDev & \\
\hline Conformity index (PTV 70 Gy) & 0.93 & 0.08 & 0.86 & 0.07 & $<0.01$ \\
\hline Conformity index (PTV 54 Gy) & 0.68 & 0.04 & 0.64 & 0.04 & $<0.01$ \\
\hline Homogeneity index (PTV 70 Gy) & 1.091 & 0.017 & 1.066 & 0.015 & $<0.01$ \\
\hline Homogeneity index (PTV 60 Gy) & 1.103 & 0.025 & 1.098 & 0.028 & 0.055 \\
\hline Homogeneity index (PTV 54 Gy) & 1.106 & 0.021 & 1.107 & 0.018 & 0.279 \\
\hline Target coverage (PTV70Gy) & 94.9 & 2.7 & 97.8 & 1.7 & $<0.01$ \\
\hline Target coverage (PTV60Gy) & 94.2 & 3.1 & 97.2 & 2.3 & $<0.01$ \\
\hline Target coverage (PTV54Gy & 93.9 & 3.0 & 95.2 & 2.7 & $<0.01$ \\
\hline Myelon maximal dose (Gy) & 42.7 & 3.6 & 41.1 & 2.6 & $<0.01$ \\
\hline Mean ipsilat. parotid dose (Gy) & 26.8 & 7.1 & 24.9 & 8.8 & $<0.01$ \\
\hline Mean contralat. parotid dose (Gy) & 21.4 & 5.3 & 19.4 & 5.9 & $<0.01$ \\
\hline Mean swallowing muscles dose (Gy) & 33.5 & 8.7 & 30.4 & 7.7 & $<0.01$ \\
\hline Mean oral mucosa dose (Gy) & 29.2 & 5.2 & 27.5 & 6.0 & $<0.01$ \\
\hline Mean mandibular bones dose (Gy) & 34.9 & 4.4 & 31.6 & 6.1 & $<0.01$ \\
\hline Mean dorsal neck tissue dose (Gy) & 28.2 & 5.0 & 24.8 & 3.7 & $<0.01$ \\
\hline
\end{tabular}

Abbreviations: PTV planning target volume

was observed for clinical plans having a mean parotid dose between 17Gy and 27Gy, see Fig. 1. In contrast, the parotid mean dose increased on average with AutoPlanning when the mean parotid dose was below 15Gy or above $35 \mathrm{~Gy}$ for the clinical plans. The parotid mean dose was reduced by more than 10 and $20 \%$ with AutoPlanning in 49 and $19 \%$ of the cases, respectively. Contrarily, the parotid mean dose was increased with
Auto-Planning by 10 and $20 \%$ in 11 and $2 \%$ of the cases, respectively (Table 3 ).

The mean dose to the swallowing muscles, oral mucosa, mandibular bones and dorsal neck tissue was significantly reduced with Auto-Planning by 3.1Gy, 1.7Gy, 3.3Gy and 3.4Gy $(p<0.01)$, respectively (see Table 2 , Figs. 2 and 3 ). In ten, seven, four and ten cases the mean dose to the swallowing muscles, oral mucosa, mandibular bones and dorsal

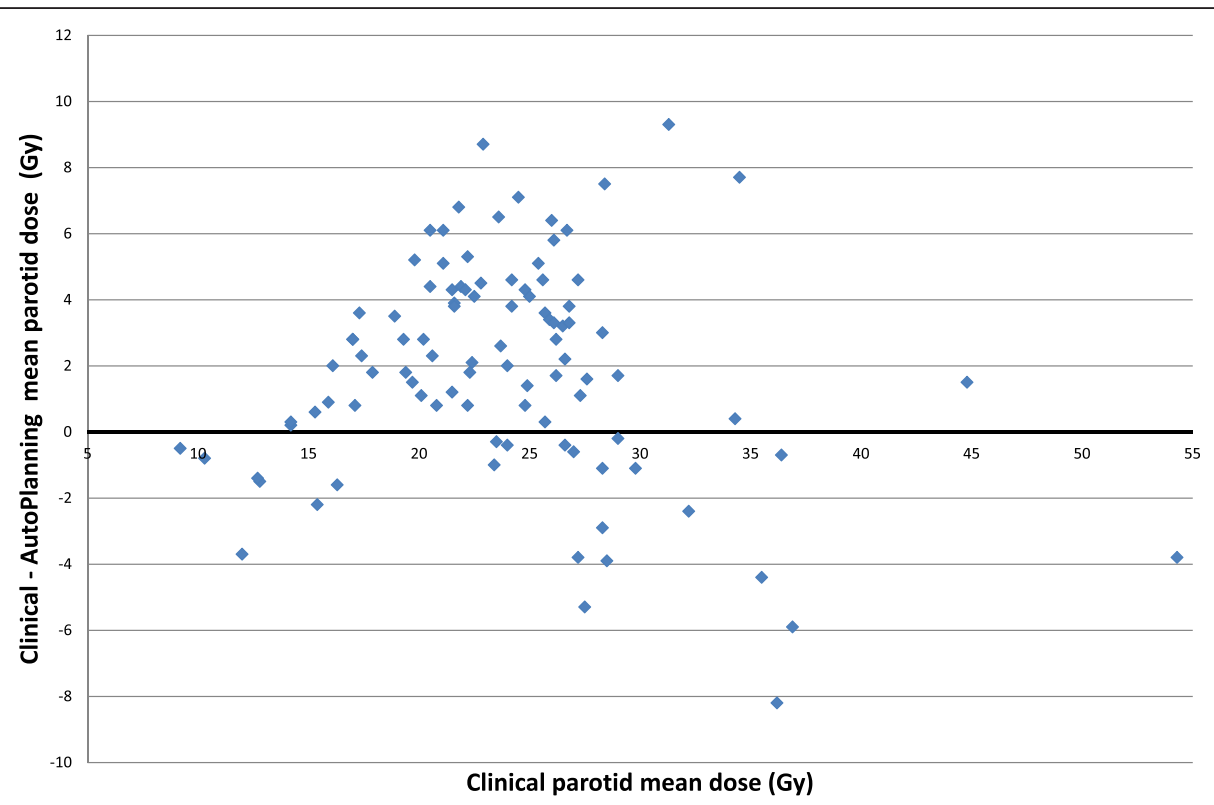

Fig. 1 Mean parotid dose difference as a function of the clinical mean parotid dose. If the values are positive, Auto-Planning reduced the parotid mean dose. If the values are negative, the parotid mean dose was reduced with the clinical plan 
Table 3 Dose difference between the clinical and the Auto-Planning plans

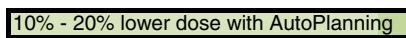

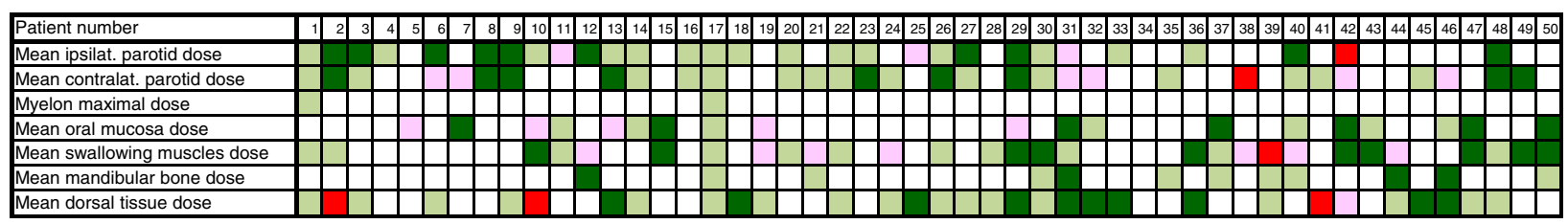

Each column represents the results for a patient. In light green, respectively dark green, Auto-Planning reduced the organ dose by more than $10 \%$, respectively $20 \%$ in comparison to the clinical plan. In pink, respectively red, the clinical plan reduced the organ dose by more than $10 \%$, respectively $20 \%$ in comparison to Auto-Planning

neck tissue was reduced by more than $20 \%$ with AutoPlanning, see Table 3. In contrast, the clinical plans showed lower mean doses to the swallowing muscles by $>20 \%$ in one case and for the dorsal neck tissue in three cases.

In 32 plans (64\%), Auto-Planning was able to reduce at least one OAR (spinal cord excluded) by more than $20 \%$. In contrast, the clinical plans showed reduced doses to at least one OAR by more than $20 \%$ in six cases (12\%), see Table 3 .

The results from the blind ranking performed by two experienced radiation oncologists showed that AutoPlanning plans were preferred in $80 \%$ of the cases and in $20 \%$ of the cases, the clinical plans were preferred.

\section{Planning time}

The effective working time required after volume definition by the clinicians to the end of the optimization, when the plan is ready to be checked by the clinicians, was measured for the last ten patients planned. $3.8 \mathrm{~min} \pm 1.1 \mathrm{~min}$ ranging from 2.45 to $6.33 \mathrm{~min}$ were required for Auto-Planning compared to $48.5 \mathrm{~min} \pm 6.0 \mathrm{~min}$ for the clinical plans ranging from 42.0 to $56.5 \mathrm{~min}$.

\section{Discussion}

In this comparative study, plans manually optimized with Eclipse were compared to plans automatically optimized with Pinnacle. Both systems are using different optimizer algorithms which potentially influence change the results. Nevertheless, planning challenges involving experienced operators have shown that both systems were able to achieve similar dose distribution $[5,6]$. Therefore, we do not believe that the results are biased due to the use of two different optimization algorithms.

Results of our study show that Pinnacle AutoPlanning, an automated iterative planning algorithm, achieved highly consistent treatment plans: target

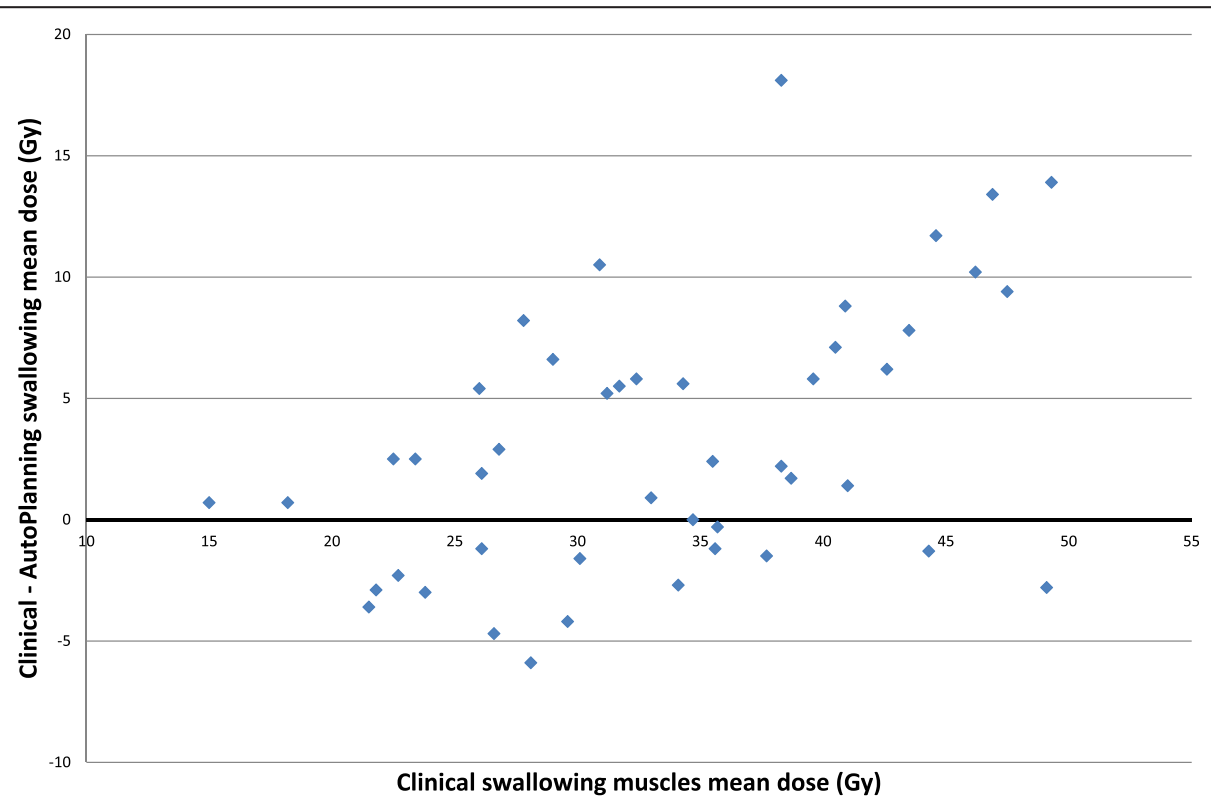

Fig. 2 Mean swallowing muscles dose difference as a function of the clinical mean swallowing muscles dose. If the values are positive, Auto-Planning had a better dose sparing of the swallowing muscles. If the values are negative, the clinical plan had a better dose sparing of the swallowing muscles 


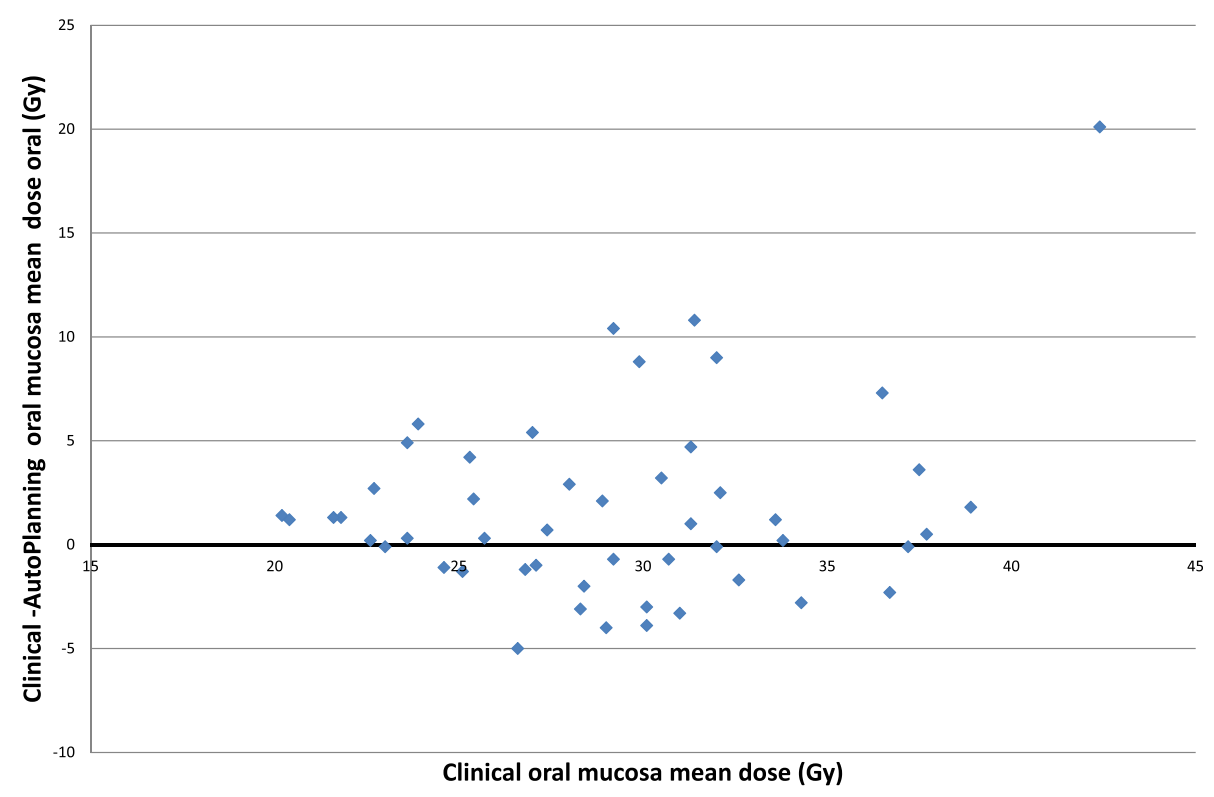

Fig. 3 Mean oral mucosa dose difference as a function of the clinical mean oral mucosa dose. If the values are positive, Auto-Planning had a better dose sparing of the s oral mucosa. If the values are negative, the clinical plan had a better dose sparing of the oral mucosa

coverage and homogeneity were significantly improved at the expense of dose conformity compared to clinically accepted historical plans. However, dose differences for all target volumes were small and are unlikely to be clinically relevant. Additionally, Auto-planning achieved a significant reduction of all OAR dose. Most importantly, Auto-Planning was able to reduce the dose to at least one OAR by more than $20 \%$ in $64 \%$ of the cases.

This finding of improved OAR sparing by a potentially clinically relevant amount could be explained by suboptimal constraints being used in the historical manual planning cohort. Plan optimization for head and neck is one of the most complex scenarios due to the complexity of the tumor shape, dose levels and location. Targets with multiple dose levels are defined in close proximity to or as overlapping OARs making the optimization a complex and time consuming process. The result will strongly depend on the skill set of the TPS Operator when setting the correct help structures, optimization objectives and constraints.

In $19 \%$ of the plans, the mean dose to one of the parotids was reduced by more than $20 \%$ with AutoPlanning. This reduction of the mean dose is expected to be clinically relevant and reduce xerostomia [7]. The normal tissue complication probability for the parotid glands reported by Eisbruch et al. is described as a continuous and monotonously increasing function of the mean dose with a steep gradient between 20Gy and 30Gy [8]. Any significant reduction of the mean dose in this range may thus be of clinical benefit. The mean parotid dose increased with Auto-Planning for clinical plans having a dose larger than 35Gy. For these cases, the targets were largely overlapping with the parotid. If this overlap is larger than 25 and $50 \%$, the priority was automatically reduced to medium or low with AutoPlanning. This could explain the higher mean parotid dose with Auto-Planning in cases where a mean dose greater than 35Gy was observed.

A significant correlation between the mean swallowing muscle dose and complications such as dysphagia has been observed [9]. A steep dose-effect relationship, with an increase of the probability of dysphagia of $19 \%$ with every additional 10Gy, was established [10]. We observed that Auto-Planning could be clinically beneficial for patients in respect to the dose delivered to the swallowing muscles. Indeed, in $20 \%$ of the cases, AutoPlanning was able to reduce the swallowing muscles mean dose by more than $20 \%$ which correspond to a dose reduction of $10.7 \mathrm{~Gy}$.

Highly consistent and improved plan quality in a relevant proportion of the cases compared to our historical plans was not only observed in DVH parameters. Indeed, radiation oncologists specialized in head and neck cancer preferred Auto-Planning plans compared to the clinical plans in $80 \%$ of the cases; however they simultaneously stated that results were close and both planning methods achieved clinically acceptable plans in all patients. This result is even more surprising as only one single Auto-Planning head and neck model-pre-set of optimization parameters-was used in Auto-planning and only one single optimization cycle was allowed. One could therefore speculate that further optimization of 
the Auto-Planning approach might be possible by 1) more customized optimization models and by 2) the use of more than one optimization cycle. By increasing the number of optimization cycles, the planner would be able to change the targets and/or OARs objectives in order to modify the dose distribution previously calculated. This would lead to a more patient specific dose distribution. Further analyses are ongoing to test these hypotheses. Besides potentially improved outcome on a patient-individual level and on an institutional level, such automated planning approaches are considered to be especially useful within the context of multi-center trials to standardize and homogenize planning and plan quality.

Other automated planning approaches have been discussed elsewhere $[9,10]$. One method is based on a previously generated site-specific plan library: the individual patient is compared to the patient population in library of plans and similarity measures are used for selection of planning optimization parameters $[2,9,11]$. This compares patient-individual DVH parameters to the database and estimates plan quality in comparison to an overall patient population. However, the application of the library is restricted to patients with identical or very similar planning objectives (number of fractions, target and OAR doses) [9]. Also, a larger number of plans are required for de-novo generation a robust library, which is challenging for smaller institutions and/or rare indications. Finally, since the knowledge of the previous plans is used to generate the objectives, the newly generated plan quality inevitably depends on the quality of the plans building the database [11]. Non-optimal plans entered in the database may degrade results with the plan library approach.

Multicriteria optimization (MCO) approaches [6-8] have been proposed for real-time assessment of the trade-off between different clinical goals. MCO allows to navigate towards a Pareto optimal plan in a database of automatically generated plans and thereby balance between targets and between organs at risk. One limitation of the MCO approach is the increased time to create the library of treatment plans before dose evaluation can be performed, although this can be overcome by faster dose calculations [12]. The MCO approach also requires significant individual user experience and interaction to determine the Pareto optimal plan, which may become especially challenging in head and neck cases were multiple targets and organs at risk then need to be balanced. In addition, if the MCO generated library of plans is fluence based, the Pareto optimal plan will not be deliverable, and further fluence segmentation is required.

\section{Conclusion}

In conclusion, our study shows that the Pinnacle AutoPlanning approach achieves highly consistent treatment plans for complex head-and-neck cancer patients: dose distributions of the target volumes were similar between the automated planning approach and manual planning. OAR sparing was significantly improved using AutoPlanning and potentially relevant improved OAR sparing was observed in more than half of the patients. Furthermore, experienced radiation oncologists preferred the dose distributions from Auto-Planning in comparison to those from the manually generated plans in $80 \%$ of the cases. Human resources required for treatment planning was substantially reduced and made independently from the experience of the treatment planner.

\section{Competing interests}

The authors declare that they have no competing interests.

\section{Authors' contributions}

JK collected the data, carried out the data analysis and drafted the manuscript. IN helped generating a model for inverse planning in Pinnacle and to collect the data. GS did the blind evaluation of the manual and automated optimized plans. MG and JK conceived the study. All authors read and approved the final manuscript.

\section{Acknowledgements}

This work has been partial supported by Philips.

\section{Author details}

'Department of Radiation Oncology, University Hospital Zurich, Klinik für Radio-Onkologie, Rämistrasse 100, CH-8091 Zürich, Switzerland. ${ }^{2}$ Philips Radiation Oncology Systems, Fitchburg, WI, USA.

Received: 9 September 2015 Accepted: 1 November 2015 Published online: 10 November 2015

\section{References}

1. Nwankwo O, Mekdash H, Sihono DSK, Wenz F, Glatting G. Knowledge-based radiation therapy (kbrt) treatment-planning versus planning by experts: validation of a kbrt algorithm for prostate cancer treatment-planning. Radiat Oncol. 2015;10(1):111.

2. Fogliata A, Wang PM, Belosi F, Clivio A, Nicolini G, Vanetti E, et al. Assessment of a model based optimization engine for volumetric modulated arc therapy for patients with advanced hepatocellular cancer. Radiat Oncol. 2014;9:236.

3. Purdie TG, Dinniwell RE, Fyles A, Sharpe MB. Automation and intensity modulated radiation therapy for individualized high-quality tangent breast treatment plans. Int J Radiat Oncol Biol Phys. 2014;90:688-95.

4. Paddick I. A simple scoring ratio to index the conformity of radiosurgical treatment plans. J Neurosurg. 2000;93:219-22.

5. Sunnuclear. Plan challenge 2015. http://planchallenge.sunnuclear.com/. 2015

6. Dosimetrists AAoM. 2014 plan challenge. http://www.medicaldosimetry.org/ pub/d98212d0-ab67-1cc2-8b4e-b7ac8f88a07f. 2014

7. Lee SH, Kim TH, Kim JY, Park SY, Pyo HR, Shin KH, et al. Evaluation of parotid gland function following intensity modulated radiation $\mathrm{t}$ herapy for head and $n$ eck cancer. Cancer Res Treat. 2006;38:84-91.

8. Eisbruch A, Ten Haken R, Kim HM, Marsh LH, Ship JA. Dose, volume, and function relationships in parotid salivary glands following conformal and intensity-modulated irradiation of head and neck cancer. Int J Radiat Oncol Biol Phys. 1999;45:577-87.

9. Deantonio L, Masini L, Brambilla M, Pia F, Krengli M. Dysphagia after definitive radiotherapy for head and neck cancer. Correlation of dosevolume parameters of the pharyngeal constrictor muscles. Strahlenter Onkol. 2013;189:230-6.

10. Levendag PC, Teguh DN, Voet P, Henri van der Est H, Noever I, de Kruijf WJM, et al. Dysphagia disorders in patients with cancer of the oropharynx are significantly affected by the radiation therapy dose to the superior and middle constrictor muscle: a dose-effect relationship. Radiat Oncol. 2007;85:64-73. 
11. Wu B, Ricchetti F, Sanguineti G, Kazhdan M, Simari P, Jacques R, et al. Data-driven approach to generating achievable dose-volume histogram objectives in intensity-modulated radiotherapy planning. Int J Radiat Oncol Biol Phys. 2011;79:1241-47.

12. Otto K. Real-time interactive treatment planning. Phys Med Biol. 2014;59:4845-59.

Submit your next manuscript to BioMed Central and take full advantage of:

- Convenient online submission

- Thorough peer review

- No space constraints or color figure charges

- Immediate publication on acceptance

- Inclusion in PubMed, CAS, Scopus and Google Scholar

- Research which is freely available for redistribution 